\title{
Engineering Plant Resistance to Tomato Yellow Leaf Curl Thailand Virus Using a Phloem-Specific Promoter Expressing Hairpin RNA
}

\author{
Yuh Tzean, ${ }^{1}$ Ho-Hsiung Chang, ${ }^{1}$ Tsui-Chin Tu, ${ }^{1}$ Bo-Han Hou, ${ }^{1}$ Ho-Ming Chen, ${ }^{1}$ Yi-Shu Chiu, ${ }^{1}$ \\ Wei-Yi Chou, ${ }^{1}$ Li Chang, ${ }^{1}$ and Hsin-Hung Yeh ${ }^{1,2,+}$ \\ ${ }^{1}$ Agricultural Biotechnology Research Center, Academia Sinica, No. 128, Sec. 2, Academia Road, Nankang District, Taipei \\ 11529, Taiwan \\ ${ }^{2}$ Department of Plant Pathology and Microbiology, National Taiwan University, No. 1, Sec. 4, Roosevelt Rd, Da'an District, \\ Taipei 10617, Taiwan
}

Accepted 21 October 2019.

\begin{abstract}
Transgenic approaches employing RNA interference (RNAi) strategies have been successfully applied to generate desired traits in plants; however, variations between RNAi transgenic siblings and the ability to quickly apply RNAi resistance to diverse cultivars remain challenging. In this study, we assessed the promoter activity of a cauliflower mosaic virus $35 \mathrm{~S}$ promoter (35S) and a phloem-specific promoter derived from rice tungro bacilliform virus (RTBV) and their efficacy to drive RNAi against the endogenous glutamate-1-semialdehyde aminotransferase gene (GSA) that acts as a RNAi marker, through chlorophyll synthesis inhibition, and against tomato yellow leaf curl Thailand virus (TYLCTHV), a begomovirus (family Geminiviridae) reported to be the prevalent cause of tomato yellow leaf curl disease (TYLCD) in Taiwan. Transgenic Nicotiana benthamiana expressing hairpin RNA of GSA driven by either the 35S or RTBV promoter revealed that RTBV::hpGSA induced stronger silencing along the vein and more uniformed silencing phenotype among its siblings than 35S::hpGSA. Analysis of transgenic $N$. benthamiana, 35S::hpTYLCTHV, and RTBV::hpTYLCTHV revealed that, although 35S:: hpTYLCTHV generated a higher abundance of small RNA than RTBV::hpTYLCTHV, RTBV::hpTYLCTHV transgenic plants conferred better TYLCTHV resistance than 35S:: hpTYLCTHV. Grafting of wild-type (WT) scions to TYLCTHV RNAi rootstocks allowed transferable TYLCTHV resistance to the scion. A TYLCTHV-inoculation assay showed that
\end{abstract}

Yuh Tzean and Ho-Hsiung Chang contributed equally.

All NGS sequence data as raw data were submitted to the National Center for Biotechnology Information (NCBI) at the Sequence Read Archive (SRA) and is available as SRP128967. The full-length TYLCTHV DNA segments $\mathrm{A}$ and $\mathrm{B}$, used for the inoculation assay, were submitted to NCBI and are available as MN495605 (DNA-A) and MN495606 (DNA-B).

${ }^{\dagger}$ Corresponding author: H.-H. Yeh; hyeh@ sinica.edu.tw

Funding: This research was funded by the Innovative Translational Agricultural Research Program.

*The $\boldsymbol{e}$-Xtra logo stands for "electronic extra" and indicates that six supplementary figures and six supplementary tables are published online.

The author(s) declare no conflict of interest.

๑) 2020 The American Phytopathological Society noninfected WT scions were only observed when grafted to RTBV::hpTYLCTHV rootstocks but not 35S::hpTYLCTHV nor WT rootstocks. Together, our findings demonstrate an approach that may be widely applied to efficiently confer TYLCD resistance.

Keywords: constitutive promoter, phloem-specific promoter, RNA interference, tomato yellow leaf curl disease, transgenic plant engineering

Biotechnology provides a means through which to improve various traits of horticultural crops in the face of major challenges such as biotic and abiotic stresses. In the genetic engineering of plants, a wide range of promoter elements have been used to drive the expression of various genes of interest (Dutt et al. 2014). Promoters not only dominate the degree of gene expression but also determine the type of tissue in which the genes are expressed. Growing evidence indicates that engineering plants with desired traits is not achieved simply through overexpressing a gene but requires the expression to be in the correct tissue (Kallolimath et al. 2018). Therefore, more understanding of how different promoters drive the spatial expression of transgenes of interest to shape the desired trait would allow for tailored precision engineering.

Constitutive promoters are thought to direct gene expression ubiquitously throughout plant development and confer high levels of transgene expression when transferred to plant cells (Dutt et al. 2014). The cauliflower mosaic virus 35S (CaMV $35 \mathrm{~S}$ ) promoter is one of the most widely used constitutive promoters in plant transformation (Porto et al. 2014). The $35 \mathrm{~S}$ promoter has been extensively used in horticultural crops for improving abiotic and biotic stress tolerance and quality traits (Dutt et al. 2014). However, even though the 35S promoter is capable of conferring high gene expression levels in most cells when transferred to plants (Kay et al. 1987), transgenic plants with $35 \mathrm{~S}$ promoter did not confer the most desired traits compared with transgenic plants that use other promoters (Dutt et al. 2012; Yin and Beachy 1995). Thus, the evaluation and selection of promoter driving the transgene of interest is especially important in plant genetic engineering.

Transgenic approaches employing RNA interference (RNAi) have been widely used to generate desired traits for crop improvement. The general strategy includes transforming plants with virus genome sequences and, subsequently, screening for 
plants that can induce RNAi against virus infection. In plants, RNAi is triggered by processing double strand RNA into small RNA (sRNA) of 20 to 24 nucleotides (nt) by Dicer-like proteins (Axtell 2013; MacRae and Doudna 2007). These sRNAs can guide a protein complex comprised of Argonaute proteins to target specific long RNA to mediate post-transcriptional gene silencing (PTGS) (Meister and Tuschl 2004) or transcriptional gene silencing (TGS) through RNA-directed DNA or histone methylation (Axtell 2013; Brodersen and Voinnet 2006). The PTGS is mainly triggered by 20- to 22-nt RNAs, while 23- to 24-nt RNA generally mediates TGS (Axtell 2013). A common problem with application of RNAi in gene silencing is that the silencing efficacy may vary between individual transgenic lines and the RNAi efficacy may even vary between progenies of the same line (Meza et al. 2001; Wang et al. 2005).

Tomato yellow leaf curl disease (TYLCD) has a devastating effect on tomato (Solanum lycopersicum) in tropical and subtropical regions globally. This disease is caused by related begomoviruses within the family Geminiviridae and leads to yield loss of up to $100 \%$ in severely affected areas (Abhary et al. 2007). The viral transmission of TYLCD is mainly by the members of the Bemisia tabaci cryptic species complex, and infection is largely associated with the phloem tissue (Ghanim 2014). Transgenic plants with ubiquitous promoters to express sRNA have been successfully applied to protect plants from TYLCD-causing begomoviruses (Ben Tamarzizt et al. 2009; Fuentes et al. 2006; Kunik et al. 1994; Lin et al. 2012; Praveen et al. 2005; Yang et al. 2014). However, the efficacy of different promoters in driving sRNA in transgenic plants has not been evaluated. Moreover, applying such a strategy to generate RNAi-mediated resistant transgenic plants of the diverse and numerous commercial tomato cultivars would require multiple transformation and screening processes, which are laborintensive and time-consuming.

Grafting experiments using mutants defective in sRNA biogenesis in either source or recipient tissue demonstrated that sRNA can move across the graft union (Molnar et al. 2010). It is considered that the movement of the systemic silencing signal is transmitted through the plant vascular phloem that serves as a connector of long-distance transport between the root and shoot systems (Mermigka et al. 2016; Palauqui et al. 1997; Voinnet and Baulcombe 1997). This graft-transmissible RNAi (gtRNAi) approach, which utilizes transgenic RNAi plants as rootstock and nontransgenic wild-type (WT) plants as scion, has been successfully applied to protect plants against tobamoviruses, potato spindle tuber viroid, and prunus necrotic ringspot virus (Ali et al. 2013; Kasai et al. 2013). The utilization of RNAi plants as rootstocks and nontransgenic scions of different cultivars may provide an efficient means to introduce TYLCDcausing begomovirus resistance to different cultivars. However, a gtRNAi approach for protecting plants from TYLCD has not yet been reported.

In this study, we compared the promoter activity of the ubiquitous $35 \mathrm{~S}$ promoter and the phloem-specific promoter derived from rice tungro bacilliform virus (RTBV). We analyzed the RNAi that targeted to the glutamate-1-semialdehyde aminotransferase (GSA) gene and tomato yellow leaf curl Thailand virus (TYLCTHV), a prevalent virus causing TYLCD in Taiwan (Jan et al. 2007; Shih et al. 2010; Tsai et al. 2011), in transgenic plants with a $35 \mathrm{~S}$ promoter or a RTBV phloemspecific promoter expressing GSA or TYLCTHV hairpin RNA (hpRNA).

Our results revealed that a transgenic plant with RTBV promoter is better than $35 \mathrm{~S}$ promoter in inducing the GSA RNAi and gtRNAi silencing phenotype. Our sRNA sequencing also revealed that even though less TYLCTHV sRNA was generated in transgenic plants with RTBV phloem-specific promoter than with $35 \mathrm{~S}$ promoter, transgenic plants with RTBV promoter triggered TYLCTHV RNAi that conferred better resistance to TYLCTHV in the regular RNAi approach. In a gtRNAi approach, noninfected WT scions were only observed when grafted on RTBV::hpTYLCTHV transgenic rootstocks but not 35S::hpTYLCTHV nor WT rootstocks. The results revealed a tailored approach that can be applied to efficiently confer TYLCD resistance by RNAi approaches.

\section{RESULTS}

\section{The CaMV 35S promoter confers stronger activity than the RTBV promoter.}

We first generated pGreen35S-Luc and pGreenRT-Luc to analyze the activity of the CaMV $35 \mathrm{~S}$ promoter (Karimi et al. 2002) and the RTBV phloem-specific promoter (Yin and Beachy 1995), by using the luciferase gene as a reporter gene downstream to the respective promoters (Supplementary Fig. $\mathrm{S} 1 \mathrm{~A}$ ). The nopoline synthase (NOS) promoter-driven Renilla luciferase gene was also inserted in pGreen35S-Luc and pGreenRT-Luc as an internal control for normalization to analyze the CaMV $35 \mathrm{~S}$ and RTBV promoters. The promoter activity assay revealed that CaMV $35 \mathrm{~S}$ promoter activity was 8.8-fold greater than that of the RTBV phloem-specific promoter (Supplementary Fig. S1B).

\section{RTBV promoter is mainly expressed in the veins of Nicotiana benthamiana.}

To confirm the spatial expression of the CaMV 35S promoter and the RTBV phloem-specific promoter used in our analysis, we generated CaMV 35S and RTBV promoter-driven enhanced green fluorescence protein (eGFP) transgenic $N$. benthamiana, 35S::eGFP and RTBV::eGFP, respectively (Fig. 1A). Green fluorescence was observed on all leaf tissues in 35S::eGFP transgenic plants (Fig. 1B), whereas green fluorescence was mainly observed in the veins of the RTBV::eGFP transgenic $N$. benthamiana (Fig. 1B). These results confirmed the spatial expression of the promoter constructs in our system and are consistent with a previous report that RTBV promoter is mainly expressed in the phloem of rice (Yin and Beachy 1995).

\section{RTBV promoter conferred more uniform silencing of GSA than CaMV 35S promoter.}

To assess the silencing phenotypes of CaMV 35S and RTBV phloem-specific promoter, we generated $N$. benthamiana expressing hpRNA of GSA driven by either 35S (35S::hpGSA) or RTBV (RTBV::hpGSA) promoters (Fig. 2A). In our study, $38 \%$ (3 of 8 ) and $47 \%$ (8 of 17) of 35S::hpGSA and RTBV:: hpGSA T0 transgenic plants, respectively, showed the GSAsilencing phenotype. Interestingly, we noticed that the GSAsilencing phenotype of the transgenic T0 N. benthamiana was more uniform in the RTBV::hpGSA leaves while 35S::hpGSA T0 transgenic plants showed varied discoloration (Fig. 2B).

The seeds from T0 plants of 35S::hpGSA-transformed $N$. benthamiana (35S03) and RTBV::hpGSA-transformed $N$. benthamiana (RT03) that showed a prominent GSAsilencing phenotype were collected for subsequent analysis. The GSA-silencing phenotype of $\mathrm{T} 1$ progeny plants (surviving on antibiotic-selection medium) of RTBV::hpGSA was consistent with their T0 parents, which exhibited uniform discoloration of the vascular tissues between plants and leaves within the individual plants (Fig. 3A). In comparison, T1 progeny plants of 35S::hpGSA-transformed parents displayed varied GSA-silencing phenotypes among individual plants and even among the leaves of the same plant (Fig. 3A). In order to evaluate the GSA messenger RNA (mRNA) level in these T1 lines, we randomly selected six $\mathrm{T} 135 \mathrm{~S} 03$ transgenic plants and 
six T1 RT03 transgenic plants for quantitative analysis of GSA mRNA by real-time quantitative reverse transcription-PCR (qRT-PCR) analysis. The average GSA mRNA of transgenic 35S::hpGSA plants was $35 \%$ of the WT plant and the average GSA mRNA of transgenic RTBV::hpGSA was $13 \%$ of the WT plant (Fig. 3B). Consistent with the observed silencing phenotype, GSA mRNA level varied among individual T1 plants derived from line $35 \mathrm{~S} 03$ of $35 \mathrm{~S}::$ hpGSA and was less variable across the six T1 individual plants of the RT03 line of RTBV:: hpGSA, as standard deviation between different siblings for 35S::hpGSA was 0.40 whereas RTBV::hpGSA was 0.17 (Fig. 3B). In addition, we also assayed for chlorophyll content for which GSA serves as an important enzyme in the biosynthetic pathway. The chlorophyll content corresponded to the GSA expression analysis and significantly lowered chlorophyll content was observed in RT03 of RTBV::hpGSA $(6,126 \mu \mathrm{g}$ per gram of leaf sample) compared with WT $(1,0214 \mu \mathrm{g}$ per gram of leaf sample) and $35 \mathrm{~S} 03$ of $35 \mathrm{~S}::$ hpGSA $(8,081 \mu \mathrm{g}$ per gram of leaf sample) (Fig. 3C).

\section{RTBV::hpGSA rootstock induced} stronger GSA silencing in the grafted WT scion compared with 35S::hpGSA rootstock.

To compare silencing activity of $35 \mathrm{~S}$ to RTBV promoters against GSA in the WT scion on the transmissible RNAi by grafting, we used T1 plants of 35S03 and RT03 transgenic parents as rootstocks and grafted them with scions of WT $N$. benthamiana. The GSA-silencing phenotype was not observed on leaves of scions with rootstocks of 35S03 (0 of 24), whereas, $87 \%$ (13 of 15 ) of leaves on scions grafted on rootstock of RT03 showed the GSA-silencing phenotype (Fig. 4A).
Real-time qRT-PCR of GSA mRNA expression levels revealed that GSA mRNA expression levels in scions grafted on 35S:: hpGSA and RTBV::hpGSA transgenic rootstock were both significantly lower than on WT rootstock, and GSA mRNA expression levels of the scion grafted on RTBV::hpGSA rootstock was significantly lower compared with a scion grafted on 35S::hpGSA (Fig. 4B). Less variation among siblings was observed in RTBV::hpGSA compared with $35 \mathrm{~S}:: \mathrm{hpGSA}$, as standard deviation between different siblings for 35S::hpGSA was 0.20 whereas RTBV::hpGSA was 0.11 (Fig. 4B).

\section{Higher percentage of RTBV::hpTYLCTHV transgenic $N$. benthamiana plants showed TYLCTHV resistance.}

To evaluate the efficacy of the RNAi and gtRNAi approach against TYLCTHV, we generated TYLCTHV infectious clones (Supplementary Fig. S2) for inoculation and then scored the degree of disease symptoms to evaluate resistance (Supplementary Fig. S3). We constructed p35ShpTYLCTHV and pRThpTYLCTHV, which contained the CaMV 35S promoter and the RTBV phloem-specific promoter, respectively, to drive the expression of hpRNA targeting to TYLCTHV DNA-A, which includes the 5'-Rep gene, intergenic region, and 5'-AV2 (nt position 2449-299) (Fig. 5A). p35ShpTYLCTHV and pRThpTYLCTHV were used to transform $N$. benthamiana to generate 35S::hpTYLCTHV and RTBV::hpTYLCTHV transgenic $N$. benthamiana, respectively. A total of 12 to $15 \mathrm{~T} 1$ transgenic plants (surviving on antibiotic-selection medium) derived from five randomly selected $\mathrm{T} 0$ transgenic lines of 35S::hpTYLCTHV and RTBV::hpTYLCTHV were collected for subsequent inoculation analysis. In general, TYLCTHV caused more severe disease symptoms in 35S::hpTYLCTHV

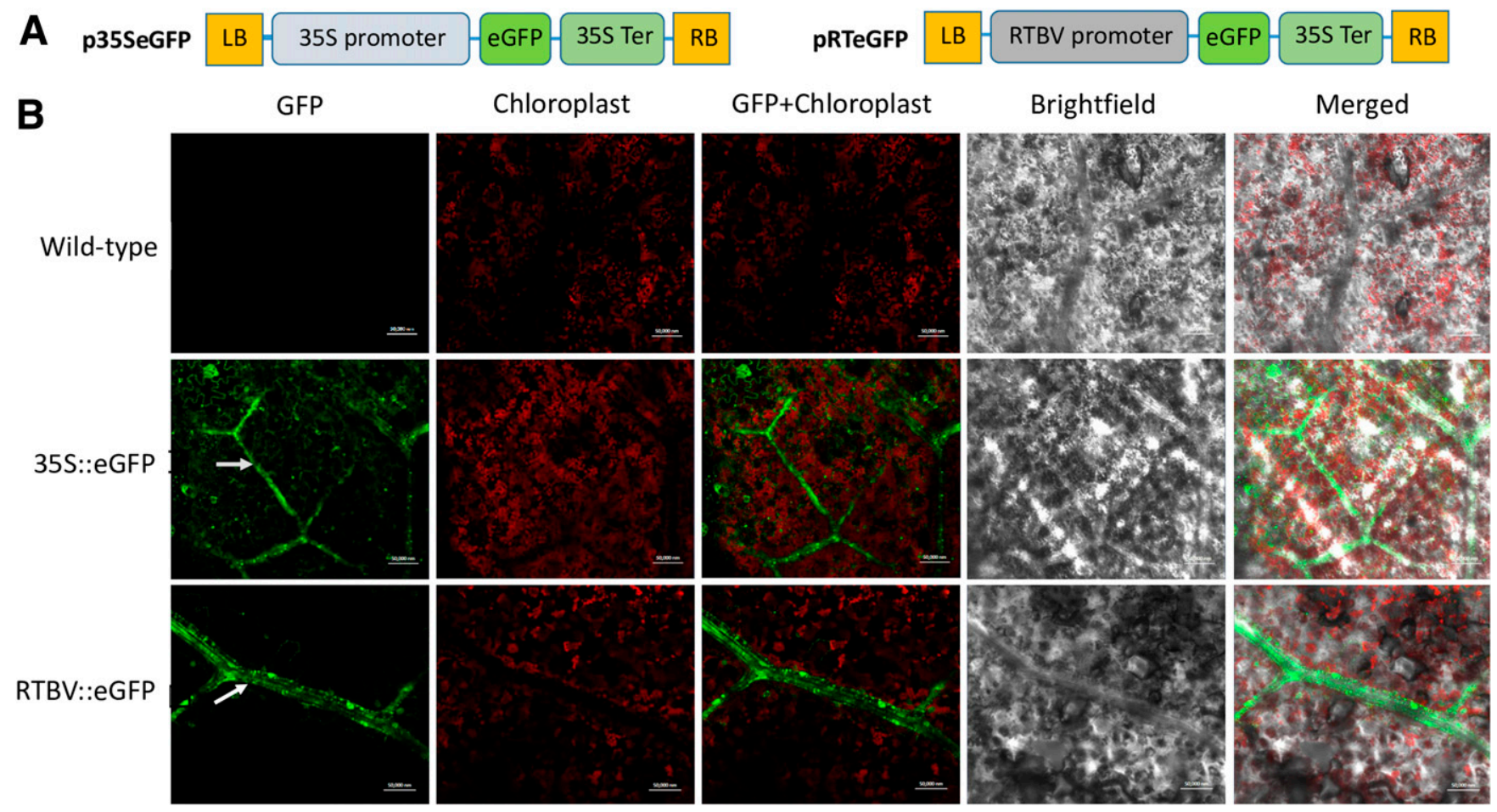

Fig. 1. Tissue expression of cauliflower mosaic virus (CaMV) 35 S promoter and rice tungro bacilliform virus (RTBV) phloem-specific promoter. A, Schematic representation of enhanced green fluorescent protein (eGFP) expression construct used to generate transgenic Nicotiana benthamiana. The eGFP gene was used to replace the cytotoxic protein gene in pK2GW7 (Karimi et al. 2002) to generate p35SeGFP. pRTeGFP was constructed by replacing the CaMV $35 \mathrm{~S}$ promoter in p35SeGFP with the RTBV phloem-specific promoter. Rectangles including T-DNA left border (LB), right border (RB), CaMV 35S terminator (35S ter) are indicated. B, p35SeGFP and pRTeGFP were used to generate transgenic $N$. benthamiana. Wild-type plants and transgenic plants were examined by use of a confocal microscope under regular light and under UV illumination with a filter to observe GFP and chloroplasts. GFP fluorescence was observed on the mesophyll and vein of plants transformed with p35SeGFP plants, GFP fluorescence was observed on the vein of plants transformed with pRTeGFP. White arrows indicate the vein of the leaf. At least two lines of each transgenic plant type were examined with similar results. 
than in RTBV::hpTYLCTHV transgenic lines (Fig. 5; Supplementary Fig. S4). The percentage of inoculated plants that were asymptomatic ranged between 0 to $29 \%$ for $35 \mathrm{~S}:$ :hpTYLCTHV and 7 to $67 \%$ for RTBV::hpTYLCTHV (Supplementary Table $\mathrm{S} 1$, experiment 1). In TYLCTHV-inoculated transgenic plants, asymptomatic plants were derived in only a single line, TY35S01, among all 35S::hpTYLCTHV transgenic lines; however, asymptomatc plants could be observed for all tested lines of RTBV::hpTYLCTHV (Fig. 5; Supplementary Table S1). The infection rate of all the tested lines of 35S: hpTYLCTHV ranged between 79 to $100 \%$ compared with 47 to $100 \%$ in RTBV::hpTYLCTHV (Supplementary Table S1, experiment 1).

T1 plants that showed the best resistance from 35S:: hpTYLCTHV (TY35S01) and RTBV::hpTYLCTHV (TYRT02) were selected for further analysis. The inoculation assay was repeated on TY35S01 and TYRT02 transgenic plants. The results showed that, although the infection rate of TYLCTHVinoculated TY35S01 and TYRT02 all reached to $100 \%, 50 \%$ of TYLCTHV-inoculated TYRT02 plants were asymptomatic, while $10 \%$ of TYLCTHV-inoculated TY35S01 were asymptomatic (Supplementary Table S1, experiment 2).

Disease severity score analysis (on a percentage scale that corresponds to the disease index) showed that the average disease severity score from two independent experiments was lower in TYRT02 plants (38.67 and 38.00\%) compared with TY35S01 plants (51.43 and $70.00 \%)$ (Supplementary Table S2). The statistical analysis of the combined total average disease severity scores from all analyzed plants showed that TYRT02 (23.00\%)

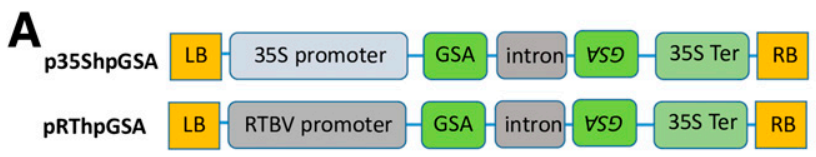

B

35S::hpGSA
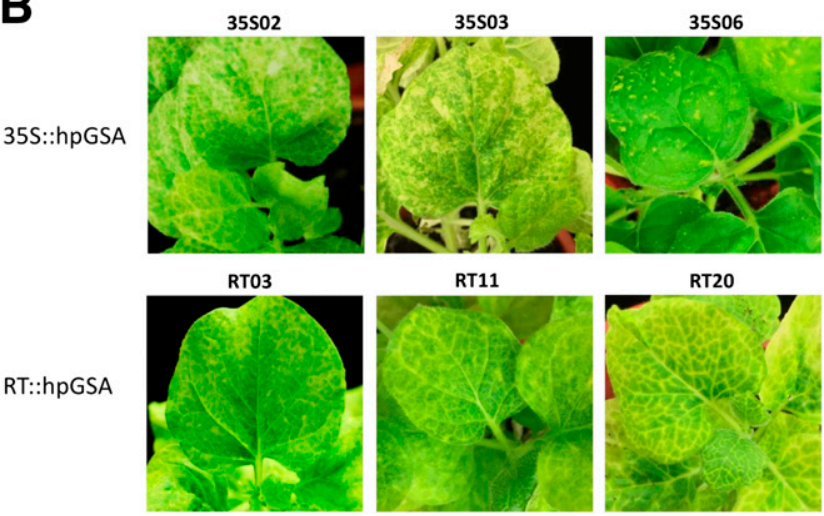

Fig. 2. Phenotypes of plants transformed with cauliflower mosaic virus (CaMV) 35S promoter and rice tungro bacilliform virus (RTBV) phloemspecific promoter to express hairpin RNA of the glutamate-1-semialdehyde aminotransferase gene (GSA). A, Schematic representation of clones used to generate GSA-silenced transgenic plants. pK2GWIWG7 (Karimi et al. 2002) contains CaMV 35 S promoter, and a partial GSA fragment was inserted in pK2GWIWG7 as an inverted repeat, to generate p35ShpGSA. RTBV phloem-specific promoter was used to replace the $35 \mathrm{~S}$ promoter in p35ShpGSA to generate pRThpGSA. Rectangles including T-DNA left border $(\mathrm{LB})$, right border $(\mathrm{RB})$, an intron region to separate the inverted repeated sequence, and CaMV $35 \mathrm{~S}$ terminator (35S Ter) are indicated. B, p35ShpGSA and pRThpGSA were used to generate 35S::hpGSA and RTBV::hpGSA transgenic Nicotiana benthamiana. Three of eight T0 lines of 35S::hpGSA plants, namely, 35S02, 35S03, and 35S06, showed the GSA-silencing phenotype. Eight of 17 T0 lines of RTBV::hpGSA plants showed the GSA-silencing phenotype. The GSA-silencing phenotype between the eight lines of RTBV::hpGSA plants was similar, and three lines, RT03, RT11 and RT20, are shown here. Plant photos were taken 3 weeks after transferring the transgenic plants from tissue culture bottles to soil. was significantly lower compared with TY35S01 (48.96\%) and WT $(86.61 \%)$ (Supplementary Table S2), and TY35S01 was significantly lower to WT (Supplementary Table S2).

TYLCTHV accumulation on TYLCTHV-inoculated WT, TY35S01, and TYRT02 transgenic plants were all analyzed in experiment 2 by real-time qPCR (Supplementary Fig. S6). TYLCTHV-infected RT02 transgenic $N$. benthamiana plants was significantly lower (56\%) compared with TYLCTHV-infected WT $N$. benthamiana (Student's $t$ test, $P<0.05$ ) and significantly lower $(61 \%)$ compared with TYLCTHV-infected 35S01 transgenic $N$. benthamiana (Student's $t$ test, $P<0.05$ ). In sum, TYRT02 performed better than TY3501 in resistance to TYLCTHV.

\section{S::hpTYLCTHV generated more viral sRNAs than RTBV::hpTYLCTHV.}

sRNA-sequence analysis was conducted on three biological plant replicates of leaf samples of TY35S01 and TYRT02 noninoculated plants. After adaptor trimming and quality filtering, short reads of 18 to $26 \mathrm{nt}$ were mapped to the sequence of TYLCTHV in the hairpin construct. The distribution of sRNAs in the sense and antisense strands of TYLCTHV sequence was similar between the transgenic lines harboring two different promoters (Fig. 6A). The amount of total sRNAs generated from the TY35S01 plant sample we sequenced was about sevenfold higher than that from the TYRT02 examined (Supplementary Table S3). Although more distinct sRNAs were recovered in the TY35S01 transgenic line (Fig. 6B; Supplementary Table S3), the unique sRNAs detected in either construct were mostly of low abundance, while the abundance of sRNAs shared between the two constructs was strongly correlated but higher in TY35S01 (Fig. 6B).

In addition, the accumulation of TYLCTHV-derived small interfering RNA (vsiRNA) in TYLCTHV-infected transgenic plants by Northern blot analysis with probes that targeted TYLCTHV DNA-A, excluding the hairpin transgenic fragment (Supplementary Fig. S6A). We pooled three biological samples corresponding to low or high virus accumulation from TYLCTHV-infected WT, TY35S01, and TYRT02 samples or mock-inoculated plants (Supplementary Fig. S5). The results showed that higher virus accumulation derived more vsiRNA fragments, which positively correlated to disease severity (Supplementary Fig. S6).

The data indicated that TY35S01 generated more sRNAs in terms of species and abundance than TYRT02, although the resistance conferred by TYRT02 was stronger than that by TY35S01 in RNAi transgenic lines.

\section{Grafting on RNAi transgenic rootstocks conferred TYLCTHV resistance in WT scions.}

T1 progeny derived from TY35S01 and TYRT02 transgenic plants that showed better TYLCTHV resistance (Fig. 5) were also used as rootstock for a grafting assay (Fig. 7). In the grafting assay, WT $N$. benthamiana scions were grafted onto T1 transgenic TY35S01 or TYRT02 rootstocks and were inoculated with TYLCTHV by agroinfiltration at 14 days after grafting. The average TYLCTHV infection rate at 21 days postinoculation on the scion grafted onto TY3501 rootstock and TYRT02 rootstock based on three independent experimental replicates were 100 and $89 \%$, respectively (Supplementary Table S4). In the TYLCTHV-inoculated scion, variation in disease severity were observed among individual scions grafted on rootstocks of WT and transgenic plants of TY35S01 or TYRT02 (Fig. 7). However, the disease severity score of TYLCTHV-inoculated scion grafted on transgenic TYRT02 was lower in all experimental sets compared with TYLCTHVinoculated scion grafted on WT and transgenic TY35S01 rootstock (Supplementary Table S5). The average disease severity score of TYLCTHV-inoculated scion was 71.82, 55.6\%, 
and $52.22 \%$ for WT, TY35S01, and TYRT02 rootstocks, respectively (Supplementary Table S5). Statistical analysis of the total average disease severity score from all analyzed plants showed that TYLCTHV-inoculated scion on TY35S01 or TYRT02 were significantly lower to WT rootstocks (Supplementary Table S5). Together, the results showed that TYLCTHV RNAi resistance can be transferred through grafting on transgenic TY35S01 or TYRT02 rootstocks. Although statistical analysis of the total average disease severity score from all analyzed plants showed no significance between TYLCTHV-inoculated scions on TY35S01 and TYRT02 rootstocks, noninfected plants were only observed on rootstock with RTBV::hpTYLCTHV but not on 35S::hpTYLCTHV.

\section{DISCUSSION}

In this study, we compared the CaMV $35 \mathrm{~S}$ promoter and a RTBV phloem-specific promoter for their promoter activity,
A WT
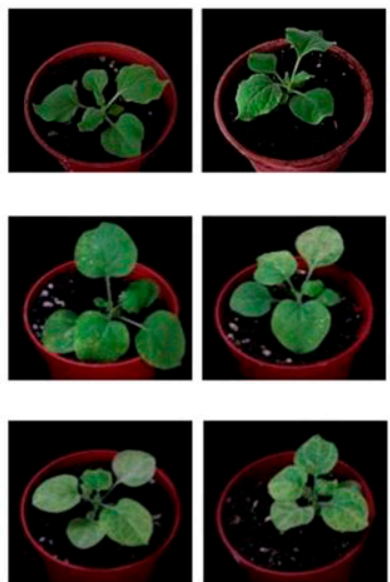
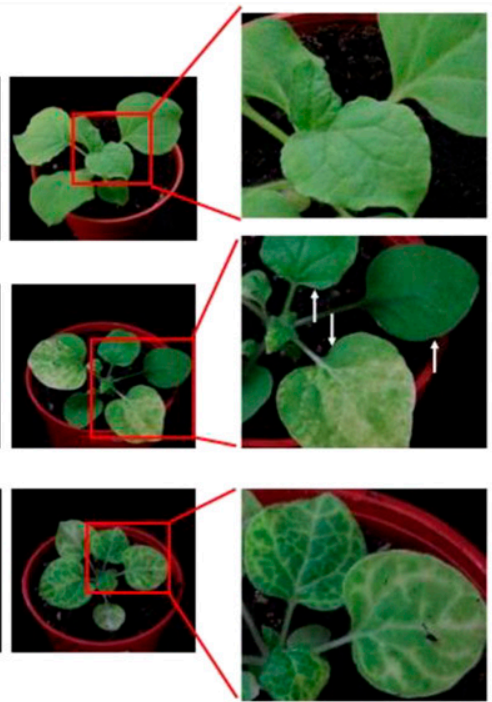

B

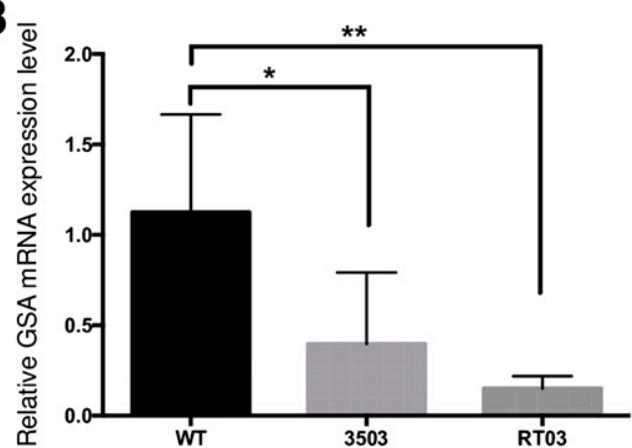

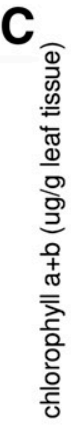

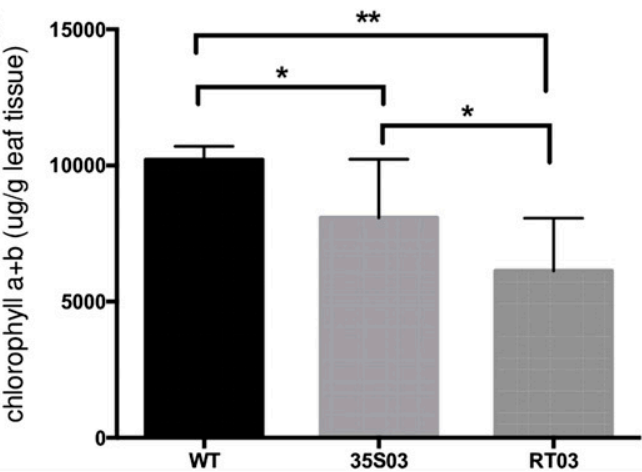

Fig. 3. Glutamate-1-semialdehyde aminotransferase (GSA) silencing phenotype and detection of GSA genes on 35S::hpGSA and RTBV::hpGSA transgenic T1 Nicotiana benthamiana. A, The T1 GSA-silencing phenotype derived from the two selected T0 plants was similar, and only progenies $35 \mathrm{~S} 03$ and RT03 are shown here. Photos were taken 4 weeks after germination. Red boxes indicate the magnified regions. White arrows indicate leaves within a $35 \mathrm{~S}:$ hpGSA plant with noticeable varied GSA-silencing phenotype. B, RNA was extracted from six randomly selected plants of wild-type $N$. benthamiana, line 35S03 of 35S:: hpGSA and line RT03 of RTBV::hpGSA for detection of GSA RNA by quantitative reverse transcription-PCR. Data represent mean \pm standard deviation (SD); $n=6$ biological replicates, two asterisks (**) indicate $P<0.01$, one (*) $P<0.05$, Student's $t$ test comparing groups as indicated. C, Chlorophyll a and b assay of $35 \mathrm{~S} 03$ of $35 \mathrm{~S}:$ hpGSA and line RT03 of RTBV::hpGSA. Data represent mean \pm SD; $n=5$ biological replicates, $* *$ indicate $P<0.01$ and $* P<0.05$, Student's $t$ test comparing groups as indicated.

A Wild-Type

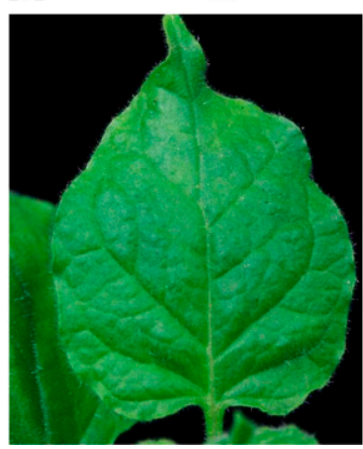

$35 S$

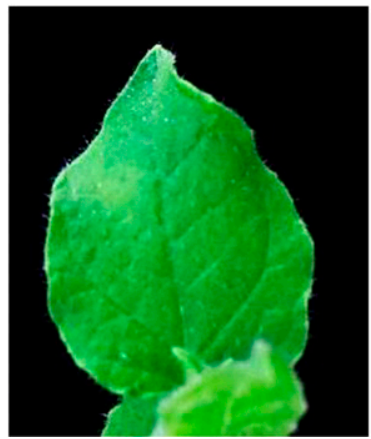

RTBV

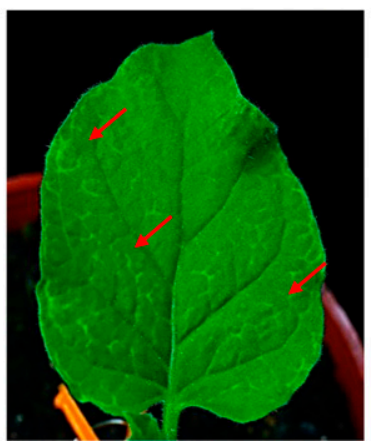

B

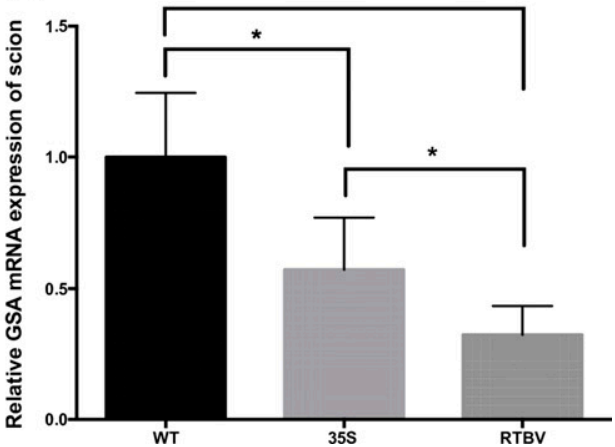

Fig. 4. Glutamate-1-semialdehyde aminotransferase (GSA) silencing phenotype and detection of GSA genes on wild-type (WT) Nicotiana benthamiana scions grafted on 35S::hpGSA and RTBV::hpGSA transgenic rootstocks. A, WT $N$. benthamiana scions were grafted on T1 transgenic plants derived from 35S:: hpGSA lines (35S02 and 35S03) and RTBV::hpGSA lines (RT03 and RT20). The silencing phenotype was observed on leaves of RTBV::hpGSA of RT03 and RT20. The phenotype of 35S02 and 35S03 and of RT03 and RT20 is similar, and only photos of line $35 \mathrm{~S} 03$ and RT03 are shown here. Photos were taken 2 weeks after grafting. Red arrows indicate the GSA-silencing phenotype. B, GSA mitochondrial RNA levels from grafted WT scion on WT, 35S::hpGSA, or RTBV::hpGSA rootstock. Data represent mean \pm standard deviation; $n=6$ biological replicates, three asterisks $(* * *)$ indicate $P<0.001$, one $(*) P<0.05$, Student's $t$ test comparing groups as indicated. 
ability to induce RNAi, and efficacy in gtRNAi. In our study, the RTBV phloem-specific promoter triggered more homogenous RNAi and gtRNAi in GSA plants compared with the CaMV $35 \mathrm{~S}$ promoter. In comparison with the CaMV 35S promoter driving TYLCTHV hpRNA, the RTBV phloemspecific promoter driving TYLCTHV hpRNA triggered stronger TYLCTHV resistance. Furthermore, we demonstrated that gtRNAi technology can be applied to protect grafted plants from TYLCTHV infection. Our results revealed several interesting phenomena that not only provide important knowledge for the application of engineering transgenic plants with RNAi technology to protect plants from TYLCD but, also, to help in designing plants with desired traits.

First, our analysis indicated that the CaMV 35S promoter has much stronger activity than the RTBV promoter, according to transient expression analysis of luciferase driven by CaMV 35S or RTBV promoters in leaves of $N$. benthamiana (Supplementary Fig. S1). Our data are in contrast to a previous report that showed that activity of RTBV phloem-specific promoter is nine times stronger than the CaMV $35 \mathrm{~S}$ promoter in leaves of transgenic rice expressing the $\beta$-glucuronidase (GUS) gene (Yin and Beachy 1995). In our study, the CaMV 35S promoter activity was 8.8-fold higher than that of the RTBV promoter (Supplementary Fig. S1). This discrepancy may result from the differences in the CaMV $35 \mathrm{~S}$ promoter sequence used in this study (Karimi et al. 2002) and with the CaMV 35S promoter used to express the GUS gene in rice (Qu et al. 1996). In the expression of the GUS gene in rice, the CaMV 35S promoter length was $351 \mathrm{bp}$ (position -343 to +8 ) (Benfey and Chua 1990), whereas the length of our promoter was $942 \mathrm{bp}$ (position -941 to +1$)($ Odell et al. 1985). Moreover, the different plant species may also account for the differences in the CaMV $35 \mathrm{~S}$ promoter activity compared with the RTBV phloem-specific promoter. Despite the discrepancy in the comparison of promoter activity, the phloem-specific expression pattern of RTBV promoter was confirmed by our analysis.

In congruence with our promoter activity assay, sRNAsequence results also indicated that transgenic $N$. benthamiana with $35 \mathrm{~S}$ promoter $(35 \mathrm{~S} 01)$ generated more sRNAs than with
RTBV promoter (TYRT02) (Fig. 6; Supplementary Table S3). Interestingly, even though the CaMV $35 \mathrm{~S}$ promoter in our study showed much stronger promoter activity than the RTBV promoter, our data revealed that the GSA-silencing phenotype was more homogenous in RTBV::hpGSA transgenic plants (Figs. $2 \mathrm{~B}$ and $3 \mathrm{~A}$ and $\mathrm{B})$.

A common problem with application of RNAi in gene silencing is that the silencing efficacy may vary between individual transgenic lines and the RNAi efficacy may even vary between progenies of the same line (Meza et al. 2001; Wang et al. 2005). This variation was also observed in 35S::hpGSA plants in this study but to a lesser extent in RTBV::hpGSA plants (Fig. 3A). Such variation in the GSA-silencing phenotype of $35 \mathrm{~S}:$ :hpGSA occurred not only to T0 and T1 plants but was also found between the leaves of the same 35S::hpGSA plant (Fig. 3A and B). We found that RTBV promoter-triggered RNAi lines showed a more prominent GSA-silencing phenotype starting from the vein, which is consistent with the phloem-specific expression of RTBV promoter. In addition, our data collected using a transgenic approach also indicated that the RTBV phloem-specific promoter performed better than the $35 \mathrm{~S}$ promoter in triggering GSA gtRNAi. Our data are consistent with previous reports in which hpGSA RNA was transiently delivered in a grafted plant. These reports also indicated that phloem-specific promoter derived from commelina yellow mottle virus (CoYMV) usually performs better than $35 \mathrm{~S}$ promoter in delivering sRNA from RNAi rootstocks to non-RNAi scions (Bai et al. 2011; Kasai et al. 2011).

Although RTBV:hpTYLCTHV generated less sRNA than 35S::hpTYLCTHV (Fig. 6; Supplementary Table S3), our analysis, as compared with different T0 transgenic lines and the siblings of the best resistance line derived from $35 \mathrm{~S}:$ : hpTYLCTHV and RTBV::hpTYLCTHV, all suggested that RTBV::hpTYLCTHV transgenic plants conferred better TYLCTHV resistance (Fig. 5; Supplementary Tables S1 and $\mathrm{S} 2$ ). This may be because sRNA expressed in RTBV:: hpTYLCTHV is mainly at the major infection site of TYLCTHV. We also demonstrated that gtRNAi can be used to protect against TYLCTHV infection (Fig. 7). This technology

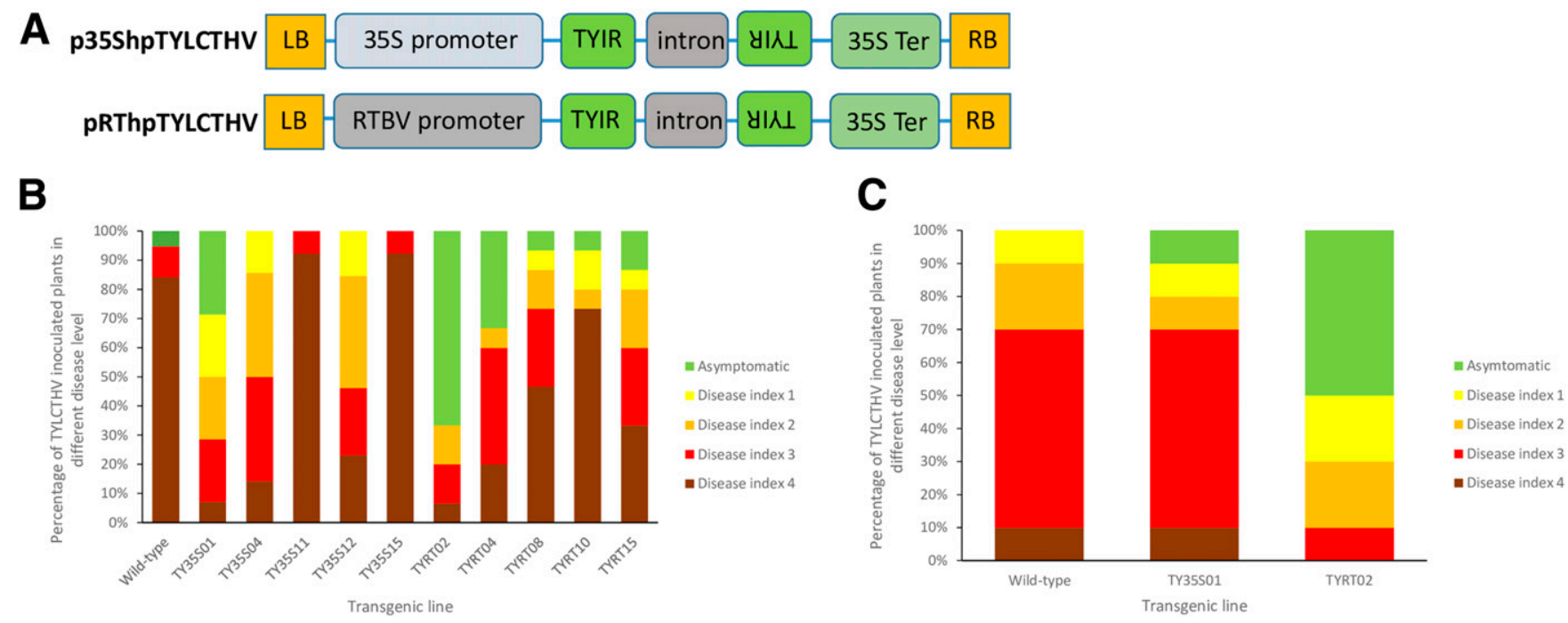

Fig. 5. Schematic representation of clones expressing hairpin RNA (hpRNA) of partial genome of tomato yellow leaf curl Thailand virus (TYLCTHV) and disease severity assay of TYLCTHV-inoculated transgenic Nicotiana benthamiana expressing hairpin TYLCTHV. A, Cauliflower mosaic virus (CaMV) 35S promoter and rice tungro bacilliform virus (RTBV) phloem-specific promoter were inserted in pK2GWIWG7 (Karimi et al. 2002) to generate p35ShpTYLCTHV and pRThpTYLCTHV to express hpRNA of a partial TYLCTHV fragment (TYIR). Rectangles including T-DNA left border (LB), right border (RB), an intron region to separate the inverted repeated of TYIR sequence, and CaMV 35S terminator (35S Ter) are indicated. B, Wild type (WT), five lines of T1 35S::hpTYLCTHV (TY35S01, TY35S04, TY35S11, TY35S12, and TY35S15), or RTBV::hpTYLCTHV (TYRT02, TYRT04, TYRT08, TYRT10, and TYRT15) were inoculated with TYLCTHV by infiltration of agrobacterium carrying the TYLCTHV infectious clone into leaves of $N$. benthamiana. C, 35S::hpTYLCTHV (TY35S01) and RTBV::hpTYLCTHV (TYRT02) that showed lower disease levels were selected for in the repeated experiment sets. 
could allow RNAi to be transferred efficiently from virusresistant RNAi rootstocks to various cultivars of WT scions without going through the time-consuming process of generating transgenic plants for each cultivar.
In the RTBV::hpGSA, an even silencing phenotype was observed on different leaves within the same transgenic plant, between $\mathrm{T} 0$ transgenic lines, or $\mathrm{T} 1$ progenies derived from the same T0 plant (Figs. 2 and 3); however, more variable TYLCTHV
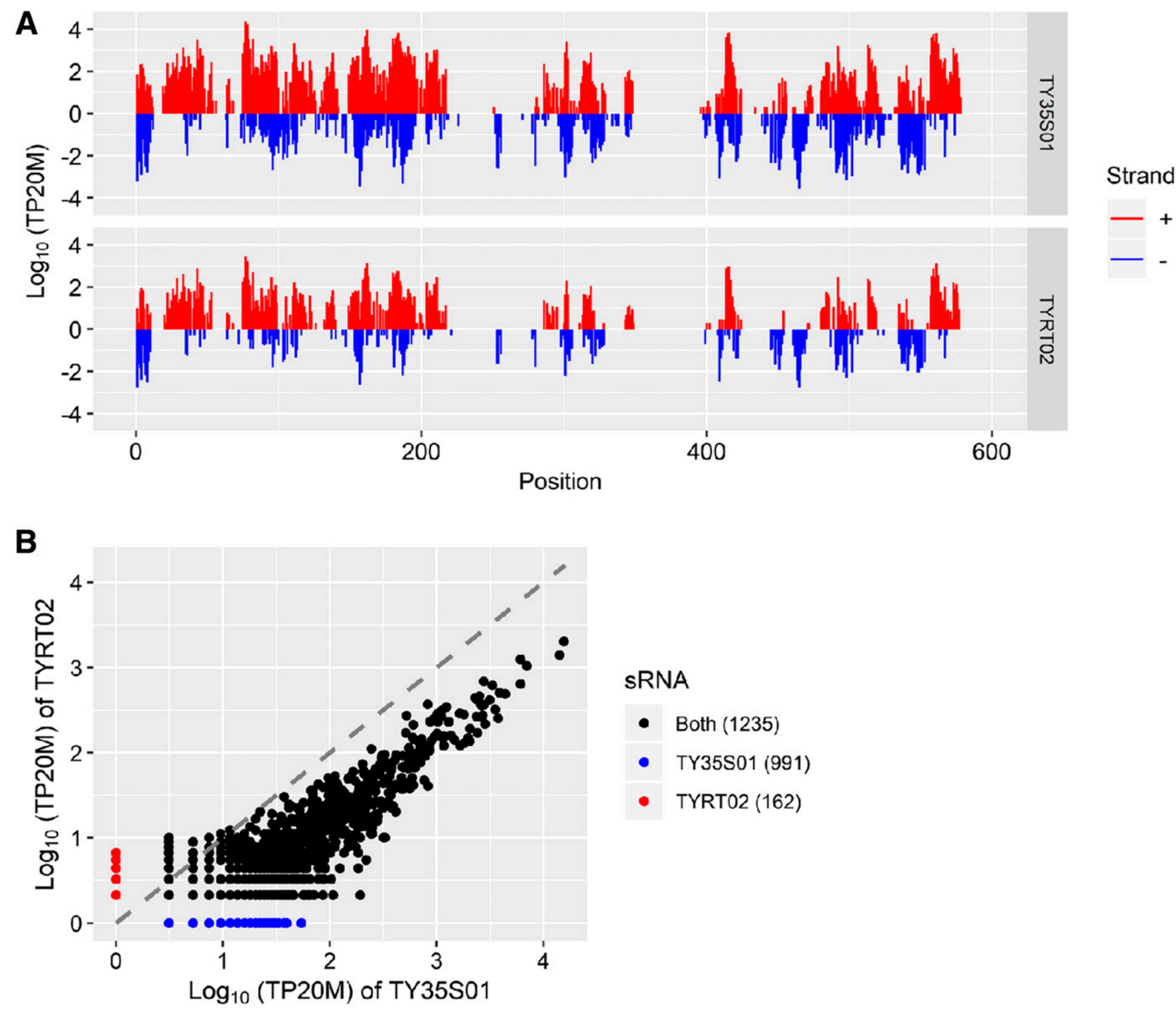

Fig. 6. Analysis of tomato yellow leaf curl Thailand virus (TYLCTHV) small RNAs (sRNA) in noninoculated transgenic RNA interference lines. A, The positional distribution of sRNAs matched to the TYLCTHV sequence. Processed sRNA sequences of 18 to 26 nt derived from 35S::hpTYLCTHV (TY35S01) and RTBV::hpTYLCTHV (TYRT02) were matched to sense and antisense sequences of TYLCTHV. Abundance of sRNA reads in each position is shown. Small RNAs matched to the sense strand are depicted in red and the antisense strand in blue. B, Scatter plot of TY35S01- and TYRT02-derived sRNAs. Sequences detected in hpRNA driven by $35 \mathrm{~S}$ and RTBV promoters are highlighted in black. Red, sequences unique to RTBV promoter-driven plants; blue, sequences unique to $35 \mathrm{~S}$ promoter-driven plants.

\section{Experiment 1}
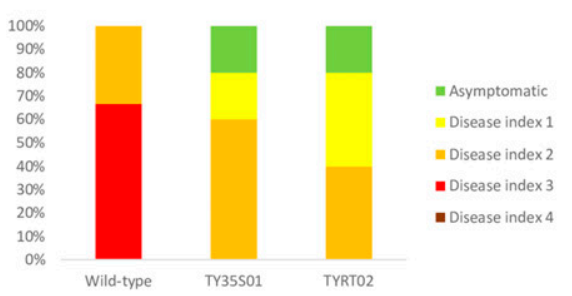

\section{Experiment 2}

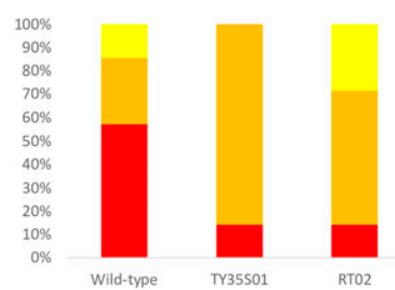

\section{Experiment 3}
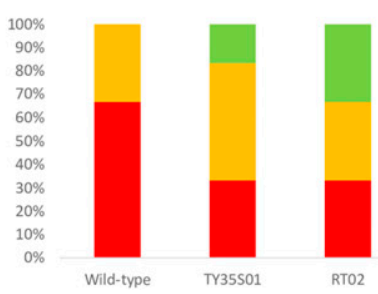

- Asymtomatic

$\because$ Disease index 1

$=$ Disease index 2

- Disease index 3

- Disease index 4

Fig. 7. Disease severity assay of tomato yellow leaf curl Thailand virus (TYLCTHV) inoculated on wild-type (WT) scion grafted on the TYLCTHV hairpinexpressing transgenic rootstock lines. Disease levels of WT scion grafted on the TYLCTHV hairpin-expressing transgenic rootstock lines (TY35S01 and TYRT02) in three independent experiment sets. 
resistance was observed in hpTYLCTHV transgenic plants (Figs. 5 and 7). For instance, the total average disease severity score in TYLCTHV-inoculated TYRT02 was significantly lower than TY35S01, and TY35S01 was significantly lower than WT (Supplementary Table S2), while statistical analysis did not resolve a significant difference between TY35S01 and TYRT02 in experiment 1 (Supplementary Table S2) or TY35S01 and WT in experiment 2 (Supplementary Table S2). However, the disease severity score of TYRT02 is consistently lower than TY35S01, and TY35S01 is consistently lower than WT in each experiment set. Moreover, the percentages of asymptomatic plants in TYLCTHV-inoculated TYRT02 plants in experiments 1 and 2 were consistently higher than TY35S01, and TY35S01 were consistently higher than WT (Supplementary Table S1). Similarly, although no statistical significance in diseases severity score was found between TYLCTHV-inoculated scions on TY35S01 and TYRT02 rootstocks, asymptomatic plants were only observed in scions grafted on TYRT02 rootstocks (Supplementary Table S4).

The plausible reason for increased variable TYLCTHV resistance observed in our TYLCTHV inoculation assay is because the outcome of TYLCTHV-infected symptoms may not solely depend on siRNA derived from our transgenic plants. It is known that, in the compatible pathogen-host interaction, when a pathogen-encoded effector accumulates to a certain level, it may suppress plant immunity (Jones and Dangl 2006). For begomovirus, it is known that proteins such as AC2, AC4, and AV2 suppress the RNAi pathway (Glick et al. 2008; Trinks et al. 2005; Vinutha et al. 2018).

Our results indicated that, compared with the $35 \mathrm{~S}$ promoter, the RTBV phloem-specific promoter triggered TYLCTHV RNAi that conferred better TLCTHV resistance in the transgenic plants. In addition, while grafting of WT scions to either $35 \mathrm{~S}$ and RTBV promoter-driven TYLCTHV RNAi rootstocks allowed transferable TYLCTHV resistance to the scion, the TYLCTHV-inoculation assay showed that noninfected WT scions were only observed when grafted to RTBV:: hpTYLCTHV rootstocks but not 35S::hpTYLCTHV nor WT rootstocks Together, these findings provide valuable knowledge about the engineering of transgenic plants and the grafting of the generated transgenic plants.

\section{MATERIALS AND METHODS}

\section{Plant materials and growth conditions.}

Transgenic $N$. benthamiana plants were generated by Agrobacterium-mediated transformation following the method described by Dandekar and Fisk (2004). T1 seeds were collected from the T0 transgenic plants. T1 seeds were screened with MS medium (Murashige and Skoog 1962) containing kanamycin. WT and transgenic plants were grown at $25^{\circ} \mathrm{C}$ under controlled light conditions $\left(200 \mu \mathrm{mol} \mathrm{m} \mathrm{s}^{-2}\right)$ with a 16 -h day and 8-h night cycle.

\section{RNA isolation and RT-PCR.}

Total RNA was extracted using TriZol reagent following the manufacturer recommendation (Invitrogen). For RT-PCR, firststrand cDNA was synthesized from $1 \mu \mathrm{g}$ of total RNA with gene-specific primers, using Moloney murine leukemia virus (Promega) RT following the manufacturer instructions. PCR amplification was performed using gene-specific oligonucleotide primer pairs. All primers used in this study are listed in Supplementary Table S6.

\section{Plant DNA isolation and real-time qPCR.}

Plant total DNA was extracted using the method described by Fulton et al. (1995). Virus accumulation was analyzed using real-time qPCR; $50 \mathrm{ng}$ of plant total DNA was added to SYBR
Premix EX Taq II reagent (Takara) for PCR reaction, with three technical repeats, and were detected by an ABI Prism 7500 sequence detection system (Applied Biosystems). The relative quantification was calculated according to manufacturer instructions (Applied Biosystems). The actin mRNA was used as an internal quantification control.

\section{Construction of vectors}

\section{for transient promoter activity analysis.}

We first replaced the CaMV 35S promoter with the NOS promoter in pGreenII-0800-Luc (Hellens et al. 2005), a dual luciferase gene expression vector. A DNA fragment (LB-fragment) was amplified by PCR, with pGreenII-0800-Luc used as template and primer pair GrFse-F/LB-NOSp-R. Another DNA fragment (NOS-fragment) was amplified by PCR, with pBI121 (Chen et al. 2003) used as template and primer pair LB-NOSp-F/NOSp-RenR. A DNA fragment (LB-NOS fragment) was amplified by PCR, with the mixed LB-fragment and NOS-fragment as templates and primer pair GrFse-F/NOSp-Ren-R. A DNA fragment (Renilla luciferase fragment) was amplified by PCR, with pGreenII-0800Luc used as template and primer pair NOSp-Ren-F/Ren-MCS-R. A DNA fragment (LB-NOS-Renilla) was amplified by PCR, with the mixed Renilla and the LB-NOS fragment as templates and primer pair GrFse-F/Ren-MCS-R. The amplified LB-NOSRenilla was digested by FseI and KpnI (New England Biolabs) and was ligated to FseI and KpnI predigested pGreenII-0800-Luc to generate pGreenNOS-Renilla.

The phloem-specific promoter sequence of RTBV (775 bp, nt position 6674-7448, accession number M65026) was directly synthesized (Purigo) as reported by Yin and Beachy (1995). PstI and SalI/SpeI restriction enzyme sites were added at the most $5^{\prime}$ and $3^{\prime}$ ends of the promoter, respectively, to facilitate the cloning. The synthesized RTBV promoter was inserted in a pGH vector (Purigo). The pGH vectors with the synthesized RTBV promoters were digested with PstI and SpeI (New England Biolabs) and were separated in a $1 \%$ agarose gel, and the RTBV promoter fragments were purified from the gel. The gel-purified RTBV promoter was cloned into a PstI- and SpeIdigested pGreenNOS-Renilla to generate pGreenRT-Luc (with RTBV promoter) vector (Supplementary Fig. S1). The cloning of pGreen35S-Luc was similar to the cloning of pGreenRT-Luc, except the gel-purified $35 \mathrm{~S}$ promoter was used, instead, to insert into the pGreenNOS-Renilla. The $35 \mathrm{~S}$ promoter was amplified by PCR, with pK2GW7 (Karimi et al. 2002) used as template and primer pair Pst-35Sp-F/Spe-35Sp-R.

\section{Construction of vectors for generating transgenic plants.}

To construct an expression vector for generating transgenic plants, pGreenRT-Luc was digested with PstI. Nucleotides $(0.1 \mathrm{mM})$ and Klenow (5 units, New England Biolabs) were added to the PstI-digested vectors ( $2 \mathrm{ug}$ ) to create blunt ends. The blunt-ended vectors were then digested with SpeI and fragments with RTBV phloem-specific promoters were separated in $1 \%$ agarose gel by electrophoresis and were then gelpurified for further ligation.

The Gateway binary vector pK2GW7 (with the $35 \mathrm{~S}$ promoter and a Gateway cloning site for overexpression of foreign genes) were digested with SacI (New England Biolabs) first and were treated with Klenow (5 units) and nucleotides $(0.1 \mathrm{mM})$ to create a blunt end. The blunt-ended pK2GW7 was then digested with SpeI to remove the $35 \mathrm{~S}$ promoter. The vectors without a $35 \mathrm{~S}$ promoter were gel-purified. The gel-purified RTBV phloem-specific promoters were then ligated to the linearized pK2GW7 (without 35S promoter) fragment to create pRTGW (with RTBV promoter).

Construction of the silencing vector with phloem-specific promoter to express hpRNA was similar to that described above except the pK2GWIWG7 vector (Karimi et al. 2002) was used, 
instead, to generate pRTGWIWG (with RTBV phloem-specific promoter).

For cloning of vectors to express eGFP, the eGFP gene was first amplified by PCR with pK7GWIWG2D (Karimi et al. 2002) template and primer pairs EGFP-F/EGFP-R. The amplified eGFP fragment was cloned into the Gateway entry vector $\mathrm{pENTR/D-TOPO} \mathrm{(Invitrogen),} \mathrm{following} \mathrm{the} \mathrm{manufac-}$ turer recommendations. The eGFP gene was then transferred into $\mathrm{pK} 2 \mathrm{GW} 7$ and pRTGW by a LR Gateway cloning reaction, to generate $\mathrm{p} 35 \mathrm{SeGFP}$ (with $35 \mathrm{~S}$ promoter) and $\mathrm{pRTeGFP}$ (with RTBV phloem-specific promoter) (Fig. 1A).

To generate GSA-silencing vectors, total RNA extracted from WT $N$. benthamiana were used as templates for RT to generate cDNA, using the primer NbGSA-R (the cDNA synthesis method is described above). The GSA fragment was amplified by PCR (521 bp in length, nt position 383-903, accession number X65974), with the cDNA templates and primer pair NbGSAF/NbGSA-R. The amplified GSA fragment was cloned into the Gateway entry vector pENTR/D-TOPO (Invitrogen) and was then transferred into pK2GWIWG7 and pRTGWIWG to generate the $\mathrm{p} 35 \mathrm{ShpGSA}$ (with $35 \mathrm{~S}$ promoter) and pRThpGSA (with RTBV phloem-specific promoter), respectively (Fig. 2A).

To generate clones expressing TYLCTHV hpRNA, total DNA was isolated from TYLCTHV-infected tomato tissue. A DNA fragment containing the $5^{\prime}$-Rep gene, intergenic region, and 5'-AV2 (designed here as TYIR; $595 \mathrm{bp}$, nt position 2449-299, accession number EF577266) was amplified by PCR with a template of the DNA purified from a TYLCTHVinfected plant and primer pair TYIR-F/TYIR-R. The amplified fragment was cloned into the Gateway entry vector pENTR/D-TOPO (Invitrogen) and was then transferred into pK2GWIWG7 and pRTGWIWG to generate p35ShpTYLCTHV and pRThpTYLCTHV, respectively.

\section{Construction of infectious clones of TYLCTHV.}

For construction of DNA infectious clones, two partially overlapped DNA-A fragments were amplified with a template of the DNA purified from TYLCTHV-infected plants and primer pairs TY-A2F/TY-A2R and TY-A3F/TY-A3R, individually. The TY-A2F/TY-A2R-amplified DNA-A fragment was digested by ApaI and BamHI (New England Biolabs) and was ligated into ApaI- and BamHI-digested pCAMBIA1390 (Hajdukiewicz et al. 1994) to produce pCAMBIA-TYA2. The TY-A3F/TY-A3R amplified DNA-A fragment was digested with BamHI and $B g l \mathrm{II}$ (New England Biolabs) and was ligated into BamHI- and BglII-digested pCAMBIA-TYA2 to generate pCAMBIA-TYAf, which contained a $1.4 \times$ partial tandem repeat DNA-A genome of TYLCTHV (Supplementary Fig. S2). The construction of the DNA-B infectious clone was similar to the construction of the DNA-A infectious clone; however, primer pairs TY-B1F/TY-B1R and TY-B2F/TY-B2R were used for the amplification of the DNA-B genome. The TY-B1F/TYB1R-amplified DNA-A fragment was cloned into ApaI- and SalI-digested (New England Biolabs) pCAMBIA1390 to generate pCAMBIA-TYB1. The TY-B2F/TY-B2R-amplified DNA-B fragment was cloned into SalI- and BglII-digested pCAMBIATYB1 to generate pCAMBIA-TYBf, which contain a $1.3 \times$ partial tandem repeat DNA-B fragment of TYLCTHV (Supplementary Fig. S2).

\section{Transient expression assay by agroinfiltration.}

pGreen35S-Luc and pGreenRT-luc were individually transformed into Agrobacterium tumefaciens LBA4404 by electroporation. A single colony of the transformed A. tumefaciens was inoculated into $5 \mathrm{ml}$ of YEP broth (per liter, $5 \mathrm{~g}$ of beef extract, $1 \mathrm{~g}$ of yeast extract, $5 \mathrm{~g}$ of peptone, $5 \mathrm{~g}$ of sucrose, $0.5 \mathrm{~g}$ of $\left.\mathrm{MgCl}_{2}\right)$ containing kanamycin $(0.1 \mathrm{~g} /$ liter $)$ and were cultured at $28^{\circ} \mathrm{C}$ with shaking $(200 \mathrm{rpm})$ overnight. A $1-\mathrm{ml}$ overnight culture was transferred into $10 \mathrm{ml}$ of fresh YEP broth with kanamycin $(0.1 \mathrm{~g} /$ liter $)$ and acetosyringone $(100 \mathrm{uM})$ and was cultured at $28^{\circ} \mathrm{C}$, with shaking, to an optical density at $600 \mathrm{~nm}$ of 1.0 , for $3 \mathrm{~h}$. The bacteria were centrifuged $(5,000 \times g)$ for $10 \mathrm{~min}$ at room temperature, were then resuspended in $10 \mathrm{ml}$ of infiltration medium (10 mM $\mathrm{MgCl}_{2}, 10 \mathrm{mM}$ 2-morpholinoethanesulfonic acid, and $100 \mathrm{uM}$ acetosyringone), and were kept at room temperature for $3 \mathrm{~h}$. The bacterial suspension was infiltrated into a leaf of $N$. benthamiana via a needleless $1-\mathrm{ml}$ syringe. After agroinfiltration, the plants were grown at $28^{\circ} \mathrm{C}$ under controlled light conditions $\left(200 \mu \mathrm{mol} \mathrm{m}^{-2} \mathrm{~s}^{-2}\right)$ with a $16-\mathrm{h}$ day and 8-h night cycle.

\section{Luciferase activity analysis.}

Luciferase activity was analyzed with the dual-luciferase reporter assay system kit (Promega), following manufacturer recommendations with some modification. Tissue samples $(0.1 \mathrm{~g})$ were collected from $N$. benthamiana leaves infiltrated with pGreen35S-Luc and pGreenRT-Luc and were ground to fine powder in liquid nitrogen. Sample powder was suspended in passive lysis buffer (200 ul) and debris were pelleted by centrifugation for $2 \mathrm{~min}$ at $10,000 \times g$ at room temperature. The supernatant was added to a luminometer plate $(20 \mu \mathrm{l}$ per well) and substrate reagent LARII (100 ul per well) was then added. Firefly luciferase activity was immediately measured by Victor2 (PerkinElmer Life Sciences). Stop \& Glo reagent was then added into the plate $(100 \mu \mathrm{l}$ per well) and Renilla luciferase activity was measured immediately. The value of firefly luciferase activity/Renilla luciferase activity was calculated.

\section{Microscope observation.}

Leaf tissue of transgenic plants with different viral promoters to express eGFP (Fig. 1) were collected and were detected for florescence signals by confocal microscope (Zeiss LSM 780, plus ELYRA S.1) with excitation at $488 \mathrm{~nm}$ and emission at 500 to $587 \mathrm{~nm}$.

\section{Quantification of chlorophyll concentration.}

The quantification of chlorophylls was as described by Warren (2008), with some modification. The leaf samples $(0.2 \mathrm{~g})$ were ground into fine powder with liquid nitrogen and were placed in a 2-ml microcentrifuge tube. Chlorophyll was extracted from the samples by adding $1.5 \mathrm{ml}$ of methanol. Samples were centrifuged for $2 \mathrm{~min}$ at $16,000 \times g$ and the supernatant was transferred to a second microcentrifuge tube. The pellet was re-extracted with a second 1.5-ml aliquot of methanol, with shaking for another $2 \mathrm{~min}$, then, centrifuged at $16,000 \times g$. The supernatant was collected to the second microcentrifuge tube and was used for measurement of chlorophyll. An amount of $200 \mu \mathrm{l}$ of chlorophyll extract was transferred into a polystyrene microplate and then detected with absorbance of A652 and A665 nm by spectrophotometer. Chlorophyll concentration was calculated using the formulae described by Warren (2008).

\section{Grafting experiments.}

Approximately 6-week-old plants were used for the cleft grafting. Transgenic $N$. benthamiana expressing hpGSA or hairpin TYLCTHV RNA were used as rootstocks. Rootstocks were prepared by first removing the shoots (maintaining four leaves on the rootstock), then making a vertical cut (about 1 to $2 \mathrm{~cm}$ ) in the center of the stem. WT $N$. benthamiana were used as scions. Scions ( 3 to $5 \mathrm{~cm}$ ) were prepared by removing all leaves except the apical leaves and trimming the base of the stem to a V shape for grafting. The grafting junction was fixed with a clip. The grafted plants were covered to avoid dehydration for 1 week. 
Virus inoculation and disease severity analysis.

For inoculation of TYLCTHV infectious clones, pCAMBIATYAf and pCAMBIA-TYBf were transferred into $A$. tumefaciens LBA4404 separately. Cultured agrobacteria containing pCAMBIATYAf or pCAMBIA-TYBf were resuspended in infiltration medium (10 mM MgCl $2,10 \mathrm{mM}$ 2-morpholinoethanesulfonic acid, and $100 \mathrm{uM}$ acetosyringone). The agrobacteria carrying pCAMBIA-TYAf or pCAMBIA-TYBf were mixed and diluted 200 times with the infiltration medium and were kept at room temperature for at least $3 \mathrm{~h}$. The mixed agrobacteria were used as inoculum to infiltrate into a leaf of transgenic $N$. benthamiana or WT $N$. benthamiana scions via a needleless 1 -ml syringe. After inoculation, the plants were grown at $28^{\circ} \mathrm{C}$ under controlled light conditions $\left(200 \mu \mathrm{mol} \mathrm{m} \mathrm{m}^{-2} \mathrm{~s}^{-2}\right)$ with a $16-\mathrm{h}$ day and 8-h night cycle. Disease symptoms of each individual plant were recorded 3 weeks postinoculation and were scored using the disease index (Supplementary Fig. S3).

\section{sRNA sequencing and data processing.}

The isolated sRNA per sample was used as input material for the sRNA library. Libraries for sequencing were generated by NEBNext Multiplex small RNA library prep set for Illumina (New England Biolabs), according to the manufacturer protocol. The libraries were pooled and sequenced on NextSeq 500. FastX-Toolkit v.0.0.14 was used for adapter trimming and quality control of sRNA reads. The trimmed reads were qualityfiltered with parameters [ $-q 30-p$ 90]. TYLCTHV sRNAs were identified by mapping the high-quality reads of 18 to $26 \mathrm{nt}$ to TYLCTHV sequence in the hairpin construct, using Bowtie 1.2.1.1 with perfect-match setting. The abundance of each sRNA was normalized to tags per 20 million (TP20M).

\section{Northern blot hybridization.}

Total RNA for Northern blot analysis of vsiRNA was extracted from leaf samples corresponding to three biological replicates of the lowest or highest TYLCTHV accumulation in WT, 35S::hpTYLCTHV, and RTBV::hpTYLCTHV. For sRNA Northern blot analysis, 3 to $5 \mu \mathrm{g}$ of total RNA was separated on $15 \%$ denaturing polyacrylamide TBE-urea gels (Invitrogen) and were transferred to Hybond-N+ membranes (GE Healthcare). Digoxigenin-labeled probes (Roche Applied Science) were synthesized with pCAMBIA-TYAf as template and primer pairs TYLCV-P-1-F and TYLCV-P-1-R, TYLCV-P-2-F and TYLCV-P-2-R, TYLCV-P-3-F and TYLCV-P-3-R, TYLCV-C1-P-F and TYLCV-C1-P-R, and TYLCV-P-5-F and TYLCV-P-5-R. The probes were hybridized to the membrane with Fast-Hyb hybridization solution (Biochain) at $37^{\circ} \mathrm{C}$ overnight. After washing three times with buffer containing $2 \times$ SSC ( $1 \times$ SSC is $0.15 \mathrm{M} \mathrm{NaCl}$ plus $0.015 \mathrm{M}$ sodium citrate)and $0.1 \%$ sodium dodecyl sulfate, hybridization signals were detected by using the chemiluminescent substrate CDP STAR (Roche Applied Science).

\section{ACKNOWLEDGMENTS}

We thank S.-C. Shen from the Confocal Microscopic Core Facility at Academia Sinica for assistance in confocal microscopy images, and L.-Y. Kuang from the Transgenic Plant Core Laboratory at Academia Sinica for assistance in generation of transgenic plants. We thank Y.-M. Shen of Taichung District Agricultural Research and Extension Station for providing the TYLCTHV isolate used in this research. We thank M. Loney of the Agricultural Biotechnology Research Center, Academia Sinica for English editing.

\section{LITERATURE CITED}

Abhary, M., Patil, B. L., and Fauquet, C. M. 2007. Molecular biodiversity, taxonomy, and nomenclature of tomato yellow leaf curl-like viruses.
Pages 85-118 in: Tomato Yellow Leaf Curl Virus Disease: Management, Molecular Biology, Breeding for Resistance. H. Czosnek, ed. Springer, Dordrecht, The Netherlands.

Ali, E. M., Kobayashi, K., Yamaoka, N., Ishikawa, M., and Nishiguchi, M. 2013. Graft transmission of RNA silencing to non-transgenic scions for conferring virus resistance in tobacco. PLoS One 8:e63257.

Axtell, M. J. 2013. Classification and comparison of small RNAs from plants. Annu. Rev. Plant Biol. 64:137-159.

Bai, S., Kasai, A., Yamada, K., Li, T., and Harada, T. 2011. A mobile signal transported over a long distance induces systemic transcriptional gene silencing in a grafted partner. J. Exp. Bot. 62:4561-4570.

Ben Tamarzizt, H., Gharsallah Chouchane, S., Lengliz, R., Maxwell, D. P., Marrakchi, M., Fakhfakh, H., and Gorsane, F. 2009. Use of tomato leaf curl virus (TYLCV) truncated Rep gene sequence to engineer TYLCV resistance in tomato plants. Acta Virol. 53:99-104.

Benfey, P. N., and Chua, N.-H. 1990. The cauliflower mosaic virus 35S promoter: Combinatorial regulation of transcription in plants. Science 250:959-966.

Brodersen, P., and Voinnet, O. 2006. The diversity of RNA silencing pathways in plants. Trends Genet. 22:268-280.

Chen, P.-Y., Wang, C.-K., Soong, S.-C., and To, K.-Y. 2003. Complete sequence of the binary vector pBI121 and its application in cloning TDNA insertion from transgenic plants. Mol. Breed. 11:287-293.

Dandekar, A. M., and Fisk, H. J. 2004. Plant transformation: Agrobacterium-mediated gene transfer. Pages 35-46 in: Transgenic Plants Methods and Protocols. L. Peña, ed. Humana Press, New York.

Dutt, M., Ananthakrishnan, G., Jaromin, M. K., Brlansky, R. H., and Grosser, J. W. 2012. Evaluation of four phloem-specific promoters in vegetative tissues of transgenic citrus plants. Tree Physiol. 32:83-93.

Dutt, M., Dhekney, S. A., Soriano, L., Kandel, R., and Grosser, J. W. 2014. Temporal and spatial control of gene expression in horticultural crops. Hortic. Res. 1:14047.

Fuentes, A., Ramos, P. L., Fiallo, E., Callard, D., Sánchez, Y., Peral, R., Rodríguez, R., and Pujol, M. 2006. Intron-hairpin RNA derived from replication associated protein $\mathrm{C} 1$ gene confers immunity to tomato yellow leaf curl virus infection in transgenic tomato plants. Transgenic Res. 15:291-304.

Fulton, T. M., Chunwongse, J., and Tanksley, S. D. 1995. Microprep protocol for extraction of DNA from tomato and other herbaceous plants. Plant Mol. Biol. Report. 13:207-209.

Ghanim, M. 2014. A review of the mechanisms and components that determine the transmission efficiency of Tomato yellow leaf curl virus (Geminiviridae; Begomovirus) by its whitefly vector. Virus Res. 186:47-54.

Glick, E., Zrachya, A., Levy, Y., Mett, A., Gidoni, D., Belausov, E., Citovsky, V., and Gafni, Y. 2008. Interaction with host SGS3 is required for suppression of RNA silencing by tomato yellow leaf curl virus V2 protein. Proc. Natl. Acad. Sci. U.S.A. 105:157-161.

Hajdukiewicz, P., Svab, Z., and Maliga, P. 1994. The small, versatile $p P Z P$ family of Agrobacterium binary vectors for plant transformation. Plant Mol. Biol. 25:989-994.

Hellens, R. P., Allan, A. C., Friel, E. N., Bolitho, K., Grafton, K., Templeton, M. D., Karunairetnam, S., Gleave, A. P., and Laing, W. A. 2005. Transient expression vectors for functional genomics, quantification of promoter activity and RNA silencing in plants. Plant Methods 1:13.

Jan, F.-J., Green, S. K., Shih, S. L., Lee, L. M., Ito, H., Kimbara, J., Hosoi, K., and Tsai, W. S. 2007. First report of Tomato yellow leaf curl Thailand virus in Taiwan. Plant Dis. 91:1363.

Jones, J. D., and Dangl, J. L. 2006. The plant immune system. Nature 444: 323-329.

Kallolimath, S., Gruber, C., Steinkellner, H., and Castilho, A. 2018. Promoter choice impacts the efficiency of plant glyco-engineering. Biotechnol. J. 13:1700380.

Karimi, M., Inzé, D., and Depicker, A. 2002. GATEWAY vectors for Agrobacterium-mediated plant transformation. Trends Plant Sci. 7:193-195.

Kasai, A., Bai, S., Li, T., and Harada, T. 2011. Graft-transmitted siRNA signal from the root induces visual manifestation of endogenous posttranscriptional gene silencing in the scion. PLoS One 6:e16895.

Kasai, A., Sano, T., and Harada, T. 2013. Scion on a stock producing siRNAs of potato spindle tuber viroid (PSTVd) attenuates accumulation of the viroid. PLoS One 8:e57736.

Kay, R., Chan, A., Daly, M., and McPherson, J. 1987. Duplication of CaMV $35 \mathrm{~S}$ promoter sequences creates a strong enhancer for plant genes. Science 236:1299-1302.

Kunik, T., Salomon, R., Zamir, D., Navot, N., Zeidan, M., Michelson, I., Gafni, Y., and Czosnek, H. 1994. Transgenic tomato plants expressing the tomato yellow leaf curl virus capsid protein are resistant to the virus. Biotechnology 12:500-504.

Lin, C.-Y., Tsai, W.-S., Ku, H.-M., and Jan, F.-J. 2012. Evaluation of DNA fragments covering the entire genome of a monopartite begomovirus for 
induction of viral resistance in transgenic plants via gene silencing. Transgenic Res. 21:231-241.

MacRae, I. J., and Doudna, J. A. 2007. Ribonuclease revisited: structural insights into ribonuclease III family enzymes. Curr. Opin. Struct. Biol. 17:138-145.

Meister, G., and Tuschl, T. 2004. Mechanisms of gene silencing by doublestranded RNA. Nature 431:343-349.

Mermigka, G., Verret, F., and Kalantidis, K. 2016. RNA silencing movement in plants. J. Integr. Plant Biol. 58:328-342.

Meza, T. J., Kamfjord, D., Håkelien, A.-M., Evans, I., Godager, L. H., Mandal, A., Jakobsen, K. S., and Aalen, R. B. 2001. The frequency of silencing in Arabidopsis thaliana varies highly between progeny of siblings and can be influenced by environmental factors. Transgenic Res. 10:53-67.

Molnar, A., Melnyk, C. W., Bassett, A., Hardcastle, T. J., Dunn, R., and Baulcombe, D. C. 2010. Small silencing RNAs in plants are mobile and direct epigenetic modification in recipient cells. Science 328:872-875.

Murashige, T., and Skoog, F. 1962. A revised medium for rapid growth and bioassays with tobacco tissue cultures. Physiol. Plant. 15:473-497.

Odell, J. T., Nagy, F., and Chua, N.-H. 1985. Identification of DNA sequences required for activity of the cauliflower mosaic virus $35 \mathrm{~S}$ promoter. Nature 313:810-812.

Palauqui, J. C., Elmayan, T., Pollien, J. M., and Vaucheret, H. 1997. Systemic acquired silencing: transgene-specific post-transcriptional silencing is transmitted by grafting from silenced stocks to nonsilenced scions. EMBO J. 16:4738-4745.

Porto, M. S., Pinheiro, M. P. N., Batista, V. G. L., dos Santos, R. C., Filho, P. A., and de Lima, L. M. 2014. Plant promoters: an approach of structure and function. Mol. Biotechnol. 56:38-49.

Praveen, S., Kushwaha, C. M., Mishra, A. K., Singh, V., Jain, R. K., and Varma, A. 2005. Engineering tomato for resistance to tomato leaf curl disease using viral rep gene sequences. Plant Cell Tiss. Org. 83:311-318.

Qu, R., de Kochko, A., Zhang, L., Marmey, P., Li, L., Tian, W., Zhang, S., Fauquet, C. M., Qu, R., de Kochko, A., Zhang, L., Marmey, P., Li, L.,
Tian, W., Zhang, S., Fauquet, C. M., and Beachy, R. N. 1996. Analysis of a large number of independent transgenic rice plans produced by the biolistic method. In Vitro Cell. Dev. Biol. Plant 32:233-240.

Shih, S. L., Tsai, W. S., Lee, L. M., Wang, J. T., Green, S. K., and Kenyon, L. 2010. First report of Tomato yellow leaf curl Thailand virus associated with pepper leaf curl disease in Taiwan. Plant Dis. 94:637.

Tashkandi, M., Ali, Z., Aljedaani, F., Shami, A., and Mahfouz, M. M. 2018. Engineering resistance against Tomato yellow leaf curl virus via the CRISPR/Cas9 system in tomato. Plant Signal. Behav. 13:e1525996.

Trinks, D., Rajeswaran, R., Shivaprasad, P. V., Akbergenov, R., Oakeley, E. J., Veluthambi, K., Hohn, T., and Pooggin, M. M. 2005. Suppression of RNA silencing by a geminivirus nuclear protein, AC2, correlates with transactivation of host genes. J. Virol. 79:2517-2527.

Tsai, W. S., Shih, S. L., Kenyon, L., Green, S. K., and Jan, F.-J. 2011. Temporal distribution and pathogenicity of the predominant tomatoinfecting begomovirus in Taiwan. Plant Pathol. 60:787-799.

Vinutha, T., Kumar, G., Garg, V., Canto, T., Palukaitis, P., Ramesh, S. V., and Praveen, S. 2018. Tomato geminivirus encoded RNAi suppressor protein, AC4 interacts with host AGO4 and precludes viral DNA methylation. Gene 678:184-195.

Voinnet, O., and Baulcombe, D. C. 1997. Systemic signalling in gene silencing. Nature 389:553.

Wang, T., Iyer, L. M., Pancholy, R., Shi, X., and Hall, T. C. 2005. Assessment of penetrance and expressivity of RNAi-mediated silencing of the Arabidopsis phytoene desaturase gene. New Phytol. 167:751-760.

Warren, C. R. 2008. Rapid measurement of chlorophylls with a microplate reader. J. Plant Nutr. 31:1321-1332.

Yang, C.-F., Chen, K.-C., Cheng, Y.-H., Raja, J. A., Huang, Y.-L., Chien, W.-C., and Yeh, S.-D. 2014. Generation of marker-free transgenic plants concurrently resistant to a DNA geminivirus and a RNA tospovirus. Sci. Rep. 4:5717.

Yin, Y., and Beachy, R. N. 1995. The regulatory regions of the rice tungro bacilliform virus promoter and interacting nuclear factors in rice (Oryza sativa L.). Plant J. 7:969-980. 